\title{
Molecular phylogenetic evidence that the Chinese viviparid genus Margarya (Gastropoda: Viviparidae) is polyphyletic
}

\author{
DU LiNa ${ }^{1}$, YANG JunXing $^{1 *}$, RINTELEN Thomas von ${ }^{2 *}$, CHEN XiaoYong ${ }^{1} \&$ \\ ALDRIDGE David ${ }^{3}$ \\ ${ }^{1}$ State Key Laboratory of Genetic Resources and Evolution, Kunming Institute of Zoology, Chinese Academy of Sciences, Kunming 650223, \\ China; \\ ${ }^{2}$ Museum für Naturkunde, Leibniz-Institut für Evolutions- und Biodiversitätsforschung an der Humboldt-Universität zu Berlin, Berlin 10115, \\ Germany; \\ ${ }^{3}$ Aquatic Ecology Group, Department of Zoology, Cambridge University, Downing Street, Cambridge CB2 3EJ, UK
}

Received February 28, 2012; accepted May 25, 2012; published online February 1, 2013

\begin{abstract}
We investigated the phylogeny of the viviparid genus Margarya, endemic to Yunnan, China, using two mitochondrial gene fragments (COI and 16S rRNA). The molecular phylogeny based on the combined dataset indicates that Margarya is polyphyletic, as two of the three well-supported clades containing species of Margarya also comprise species from other viviparid genera. In one clade, sequences of four species of Margarya even cluster indiscriminately with those of two species of Cipangopaludina, indicating that the current state of Asian viviparid taxonomy needs to be revised. Additionally, these data suggest that shell evolution in viviparids is complex, as even the large and strongly sculptured shells of Margarya, which are outstanding among Asian viviparids, can apparently be easily converted to simple smooth shells. The molecular data also indicate that the species status of the six extant species of Margarya should be re-assessed.
\end{abstract}

Margarya, molecular phylogeny, polyphyly, mtDNA China

Citation: Du L N, Yang J X, von Rintelen T, et al. Molecular phylogenetic evidence that the Chinese viviparid genus Margarya (Gastropoda: Viviparidae) is polyphyletic. Chin Sci Bull, 2013, 58: 2154-2162, doi: 10.1007/s11434-012-5632-y

The ten freshwater lakes of Yunnan, in the southwest plateau of China, host many endemic macrophytes and animals including fishes, mollusks and crustaceans [1-9]. The lakes, of which Er-hai, Dianchi, and Fuxian are the largest (Figure 1 ), were formed during the late Pliocene and early Pleistocene [10]. Today, they drain into three different river systems (compare Figure 1) [10,11].

The viviparid Margarya Nevill, 1877 is endemic of the Yunnan lakes. First discovered by the Margary expedition to Ta-li fu (modern day Lake Er-hai), Nevill (1877) set up the genus based on the type species Margarya melanioides collected from that lake $[12,13]$. Margarya has been described solely based on shell characters, viz. its large scalariform shells with a distinct suture and strong spiral sculpture

*Corresponding authors (email: yangjx@mail.kiz.ac.cn; Thomas.Rintelen@mfn-berlin.de)
(Nevill, 1877; Figure 2) which make it easily distinguishable from all other Asian viviparids [12]. While other viviparid genera found in Yunnan, notably Cipangopaludina Hannibal, 1912 and Sinotaia Haas, 1939, differ in that they are unsculptured and smaller (Sinotaia), a great degree of shell variability is also found within Margarya (Figure 2) $[14,15]$. Considerable variation in sculpture has also been observed in the type species just within Lake Dianchi (Figure 2) [16].

Till now, a total of ten Margarya species, including three fossil taxa and a recently extinct species, have been described [12,14,17-20]. The six extant species in the genus Margarya occur in eight lakes, viz Er-hai, Dianchi, Yangzonghai, Fuxian, Xingyun, Qilu lakes and Lake Yilong and Datun are $[15,19]$. With the exception of one species (Margarya yangtsunghaiensis), all extant species of Margarya 


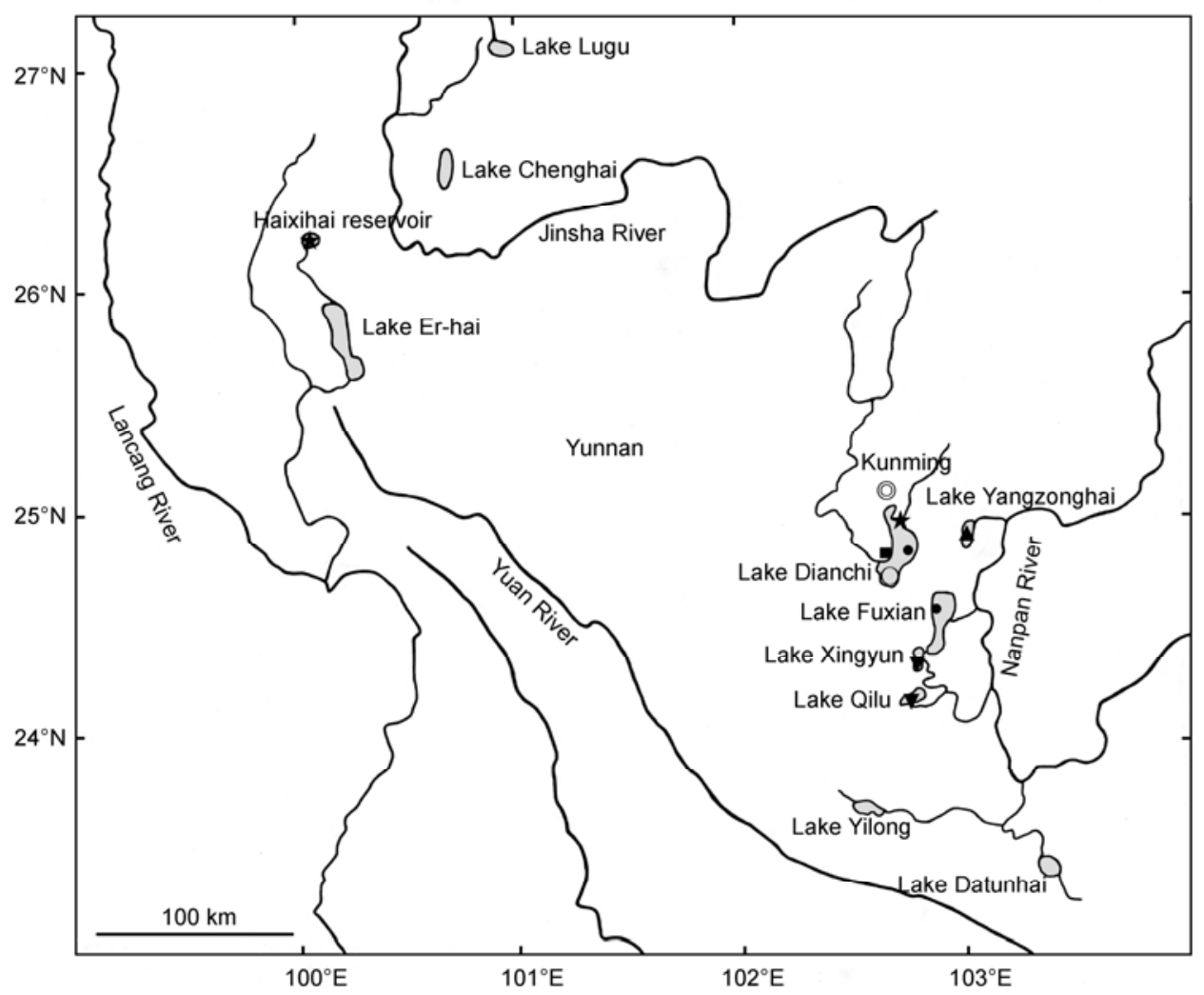

Figure 1 Map of sampling localities of each species in Yunnan Province, southwestern China. $\star$, M. melanioides; $\mathbf{\Delta}$, M. yangtsunghaiensis; $\mathbf{\nabla}$, M. mansuyi; $\bullet$, M. bicotata; $\mathbf{\bullet , M}$. monodi; $\mathrm{O}$, M. tropidophora.

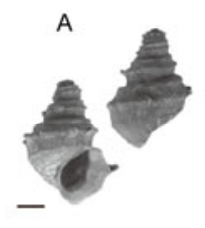

D
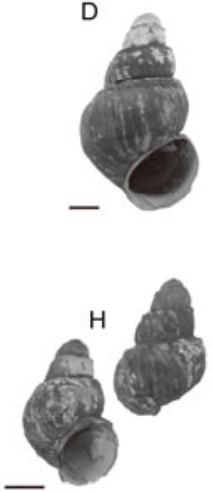
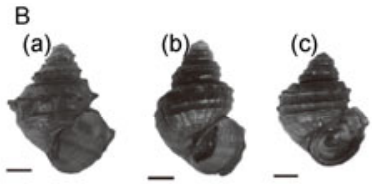

$\mathrm{E}$
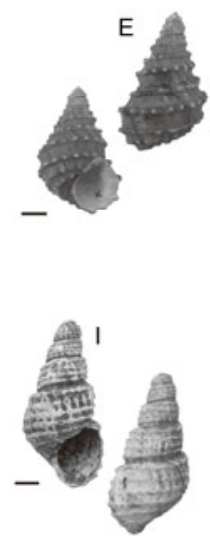
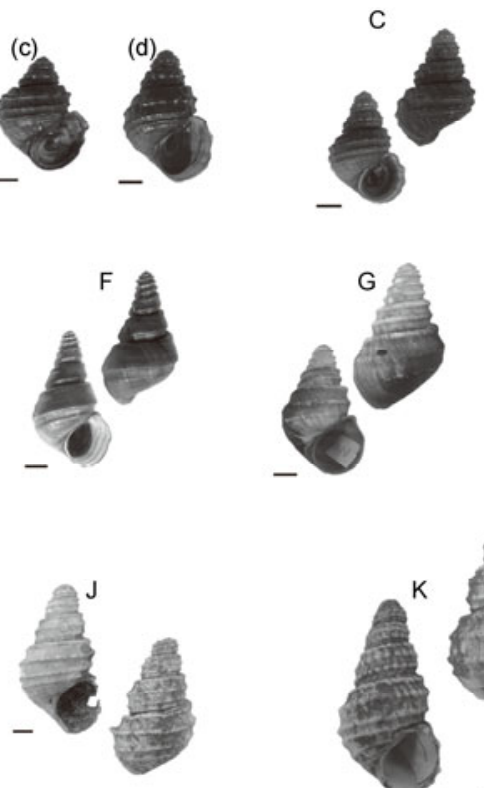

Figure 2 The shells of Margarya. A, Margarya melanioides (IOZ-FG164208), Lake Er-hai. B, Four types of Margarya melanioides. a, A type (KIZ-DA2005002); b, B type (KIZ-DLN2008002); c, C type (KIZ-45); d, D type (KIZ-DLN2008080), Lake Dianchi. C, Margarya monodi (KIZ-13), Lake Dianchi. D, Margarya tropidophora (IOZ-FG89708), Lake Er-hai. E, M. yangtsunghaiensis (holotype IOZ-00001), Lake Yangzonghai. F, Margarya mansuyi (KIZ-DLN2007008), Lake Xingyun. G, Margarya bicotata (holotype, IOZ), Lake Fuxian. H, Margarya francheti (IOZ-FG89664), Lake Dianchi. I, Margarya elongata (holotype, IOZ-FG00074), Lake Dianchi. J, Margarya yini (holotype, IOZ), Lake Dianchi. K, Margarya tchangsii (paratype, IOZ-FG89703), Lake Dianchi. Scale bar=1 cm. 
occur in two or more of these lakes [21].

The phylogenetic relationships of Margarya to other Asian viviparids entirely unclear. The genus has been described based on shell morphology only and anatomical data are still lacking. The intrageneric systematics of Margarya has also been poorly investigated. Based on shell characters, Tchang and Tsi [12] considered M. yangtsunghaiensis as the most "primitive" species in the genus Margarya. However, Chen and Zhang [22] inferred M. melanioides to be the most basal taxon based on karyotypes. Based on a Neighbor-Joining Analysis of COI sequences from a single individual of each species, Huang and Wang [23] reconstructed the phylogeny of Margarya in Dianchi Lake and suggested that Margarya in Lake Dianchi is monophyletic. However, this result is rather inconclusive because of the insufficiencies in sampling and taxon coverage.

In the present study, the phylogenetic relationships of Margarya are investigated using the mitochondrial $C O I$ and 16S rRNA genes based on an extensive sampling of Margarya. The primary aim of this study is to test the monophyly of Margarya and to uncover its phylogenetic relationship to other viviparids. We also aim to investigate the relationships of Margarya species and populations from different lakes and to explore the possibility of within-lake radiation in Lake Dianchi, which hosts four species of Margarya.

\section{Materials and methods}

\subsection{Taxon sampling}

The specimens of Margarya examined in this study were collected primarily between 2005 and 2008. All voucher specimens used in this study were deposited at the Kunming Institute of Zoology, Chinese Academy of Sciences. The identification of Margarya species followed the criteria of Tchang and Tsi [12]. While Margarya has been recorded from eight lakes, fresh samples could only be obtained from five lakes (Figure 1). Due to heavy pollution and over harvesting, M. mansuyi has disappeared from Lakes Yilong and Datun, and $M$. melanioides has disappeared from Lake Er-hai, but a few specimens of $M$. melanioides were collected from Haixihai reservoir, which forms part of Lake Er-hai. Additionally, we checked all specimens of Margar$y a$ preserved in the Invertebrate Museum of the Institute of Zoology, Beijing.

A total of 55 specimens have been sequenced, including 42 specimens of all 6 extant species of Margarya and 13 specimens of Cipangopaludina Hannibal, 1912 from the Yunnan lakes (Table 1). Sequences of other viviparid genera (Table 1) were retrieved from GenBank. Sequences of two species of the ampullariid, Pomacea bridgesii (Reeve, 1856) and $P$. canaliculata (Lamarck, 1819), and two species of Viviparinae, Viviparus ater (De Cristofori \& Jan, 1832) and $V$. contectus (Millet, 1813) from GenBank were used as outgroup to root the phylogeny [24].

\subsection{DNA extraction, PCR amplication, and sequencing}

Genomic DNA was extracted from muscle tissue fixed in 99\% ethanol. Tissue samples were digested using proteinase $\mathrm{K}$ and subsequently purified following a standard phenol/ chloroform isolation and ethanol precipitation. Two mitochondrial gene fragments (COI and $16 S$ rRNA) were amplified by polymerase chain reaction (PCR) using the primer pairs LCO1490/HCO2198 (COI) [25] and 16Sar-L/16Sbr-H (16S) [26]. PCR amplifications were conducted in a total volume of $50 \mu \mathrm{L}$ using the following cycling conditions: an initial denaturing step at $94^{\circ} \mathrm{C}$ for $10 \mathrm{~min}$, followed by 35 cycles at $94^{\circ} \mathrm{C}(1 \mathrm{~min})$, annealing at $46-50^{\circ} \mathrm{C}\left(\mathrm{COI}: 46^{\circ} \mathrm{C}\right.$

Table 1 Species used and GenBank Accession numobers

\begin{tabular}{|c|c|c|c|c|c|}
\hline Taxon & Voucher number & Locality & Country & $\mathrm{COI}$ & $16 \mathrm{~S}$ \\
\hline \multicolumn{6}{|l|}{ Outgroup } \\
\hline Pomacea bridgesii & - & GenBank & S. America & - & EU274500 \\
\hline P. canaliculata & - & GenBank & S. America & EU2744574 & EU2744501 \\
\hline Viviparus ater & - & Goldbach & Switzerland & FJ405882 & FJ405734 \\
\hline$V$. contectus & - & Hellebaek lakes & Denmark & FJ405835 & FJ405692 \\
\hline \multicolumn{6}{|l|}{ Ingroup } \\
\hline B. jeffreysi & - & River Songwe & Tanzania & FJ405857 & FJ405702 \\
\hline B. crawshayi & - & Mkolo & Zambia & FJ405867 & FJ405710 \\
\hline Neothauma tanganyicense & - & Mpulungu & Zambia & FJ405843 & FJ405709 \\
\hline Angulyagra sp. & - & Nam Dinh & Vietnam & FJ405879 & FJ405723 \\
\hline Larina sp. & - & Queensland & Australia & - & FJ405733 \\
\hline Mekongia sp. & - & Hanoi & Vietnam & FJ405875 & FJ405722 \\
\hline Sinotaia sp. & - & Hanoi & Vietnam & FJ405883 & FJ405728 \\
\hline Cipangopaludina chinensis & - & GenBank & - & FJ710298 & FJ710214 \\
\hline
\end{tabular}




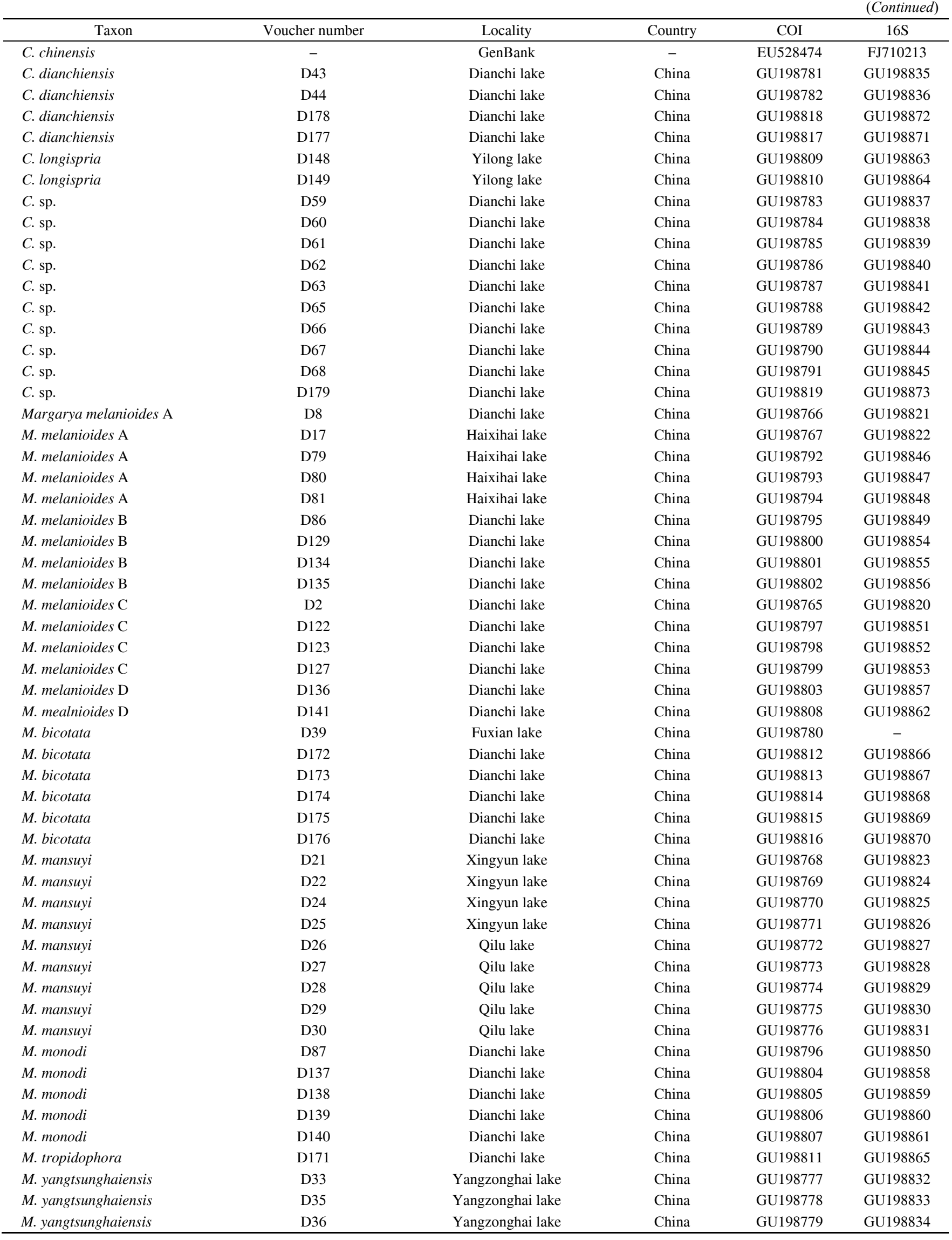


for $3 \mathrm{~min} 30 \mathrm{~s}$; $16 \mathrm{~S}$ rRNA: $50^{\circ} \mathrm{C}$ for $1 \mathrm{~min}$ ) and extension at $72^{\circ} \mathrm{C}$ ( 1 min $45 \mathrm{~s}$ for $\mathrm{COI} ; 1 \mathrm{~min}$ for $16 \mathrm{~S}$ rRNA) with a final extension step of $10 \mathrm{~min}$ at $72^{\circ} \mathrm{C}$. PCR products were purified via spin columns. Sequencing was performed directly using the corresponding PCR primers. DNA sequences of both strands were obtained using the BigDye Terminator v3.1 on an ABI PRISM 3730 following the manufacturer's instructions. All new sequences have been deposited in GenBank (Accession Nos: GU198765-GU198873).

\subsection{Phylogenetic analyses}

Sequences were aligned using Clustal X v1.83 with default gap costs and were revised by eye to maximize the homology of positions [27]. Sequences of $1148 \mathrm{bp}$ were unambiguously aligned for all taxa (641 bp of COI and $507 \mathrm{bp}$ of $16 S$ ). To explore the saturation of the sequences, transitions and transversions were plotted against sequence divergence using DAMBE (ver. 4.1.19) [28]. Prior to combining different gene fragments, we investigated the degree of heterogeneity between them using the partition-homogeneity test [29].

The data was subjected to three different phylogenetic analyses using maximum likelihood (ML), maximum parsimony (MP) and Bayesian inference (BI). ML and MP analyses were carried out with PAUP* v4.0b10 [30]. The MP method was performed using heuristic searches with 1000 random-addition sequence replicates, transitions and transversions were given equal weight. Consistency index $(\mathrm{CI})$, retention index (RI), and rescaled consistency index were calculated for the resulting MP trees. ML analysis was performed using heuristic searches with 10 random-addition sequence replicates, based on the best substitution model, which was selected using the Akaike information criterion (AIC) [31], as implemented in the computer program Modeltest v3.7 [32]. Support for nodes of the resulting MP and ML trees was assessed by analyses of 1000 and 100 bootstrap replicates in PAUP* v4.0b10, respectively [33]. Clades with bootstrap values and posterior probabilities (see below) above 95 were considered to be well-supported. Finally, BI was carried out using MrBayes v3.1.2 based on the best substitution model [34]. To reduce the risk of the chain being trapped on local optima $[35,36]$, two runs were performed simultaneously with four Markov chains (three heated and one cold), starting from random trees. The Markov chains were run for 2000000 generations, and sampling every 100 generations thinned the data to 20000 sample points. The program Tracer v1.3 was used to determine when the $\log$ likelihood $(\ln L)$ of sampled trees reached a stationary distribution [37]. Generations sampled before the chain reached stationary were discarded as burn-in, and the remaining trees were used to create $50 \%$ majority-rule consensus tree and to estimate Bayesian posterior probabilities (BPPs). The average $p$-distances of COI and $16 S r R N A$ sequences between major clades were calculated using the program MEGA v3.1 [38].

\section{Results}

\subsection{Sequence and tree statistics}

The sequence data we obtained did not reach saturation. Of the $1148 \mathrm{bp}$ in the combined alignment, 489 positions were variable and 386 were phylogenetically informative $(42.6 \%$ and $33.6 \%$, respectively), and among 386 informative positions, 196 and 190 characters were contributed by $C O I$ and $16 \mathrm{~S}$, respectively. Genetic distances ( $p$-distance) for both genes are shown in Tables 2 and 3.

The GTR+G+I model was selected as the best-fitting model of nucleotide substitution. Settings for this model were as follows: $R$-matrix $=(0.3434,8.9517,1.3819$, $0.5061,3.9593$ and 1.0000 $)$; base frequencies $=(\mathrm{A}=0.2961$, $\mathrm{C}=0.1199, \mathrm{G}=0.1796$ and $\mathrm{T}=0.4044)$; Proportion of invariable sites $=0.2924$; and the shape-parameter of the $\gamma$ distribution $=0.6243$.

The twelve most parsimonious trees (MPTs) were obtained with 1132 evolutionary steps, a consistency index (CI) of 0.593 and a retention index (RI) of 0.849 . The likelihood value of the ML tree was $\ln L=-7043.23$, and the likelihood values of the consensus tree in the Bayesian approach were -6925.65 and -6937.17 for the cold chain of runs 1 and 2, respectively. The first 1000 samples were discarded as burn-in and the remaining trees were used to construct the consensus tree.

Table 2 Genetic distances (uncorrected $p$-distances) for $16 \mathrm{~S}$ rRNA ${ }^{\mathrm{a}}$ )

\begin{tabular}{|c|c|c|c|c|c|c|c|}
\hline & Myan & Mbic & Mman & Mekong & $\mathrm{Cch}$ & Clo & Mdian \\
\hline Myan & $0.004-0.012$ & & & & & & \\
\hline Mbic & 0.129 & - & & & & & \\
\hline Mman & 0.118 & 0.006 & $0-0.107$ & & & & \\
\hline Mekong & 0.119 & 0.045 & 0.029 & 0.020 & & & \\
\hline Cch & 0.108 & 0.099 & 0.087 & 0.108 & 0 & & \\
\hline Clo & 0.102 & 0.093 & 0.077 & 0.092 & 0.033 & 0 & \\
\hline Mdian & 0.095 & 0.090 & 0.076 & 0.091 & 0.035 & 0.006 & $0-0.016$ \\
\hline
\end{tabular}

a) Myan, Margarya yangtsunghaiensis; Mbic, Margarya bicotata; Mman, Margarya mansuyi; Cch, Cipangopaludina chinensis; Clo, Cipangopaludina longispria; 'Mdian' was shared by species from Lake Dianchi and Haixihai reservoir; Mekong, Mekongia sp. 
Table 3 Genetic distances (uncorrected $p$-distances) for COI

\begin{tabular}{lccccc}
\hline & Myan & Mbic & Mman & Mekong & \\
Myan & $0.006-0.010$ & & & \\
Mbic & 0.1565 & - & & \\
Mman & 0.1541 & 0.0158 & $0-0.009$ & & \\
Mekong & 0.1629 & 0.0462 & 0.0449 & 0.008 \\
Cch & 0.1574 & 0.1105 & 0.1125 & 0.1122 & 0.0602 \\
Clo & 0.1448 & 0.1100 & 0.1091 & 0.1139 & 0.006 \\
Mdian & 0.1408 & 0.1100 & 0.1100 & 0.1150 & 0.0645 \\
\hline
\end{tabular}

The partition-homogeneity test indicates no significant heterogeneity among the two gene fragments ( $P$-value is 0.265). Therefore, the combination of the COI and $16 \mathrm{~S}$ gene partitions should not be an issue.

\subsection{Phylogenetic relationships}

Three phylogenetic analyses of the combined dataset yielded identical topologies. Thus, only the BI tree is presented here (Figure 3). The following relationships were revealed by the three analyses:

The monophyly of Margarya is not supported, with species being found in three different clades (clades 1-3, Figure 3 ) that with one exception (Clade 1) also comprise representatives of other viviparid genera. The species of Margarya in these clades are also set apart by comparatively large genetic distances of at least $7.7 \%(16 S)$ and $11.0 \%$ (COI; Tables 2 and 3).

Margarya yangtsunghaiensis (Clade 1) is basal and sister taxon to a clade comprising the remaining Margarya species plus Cipangopaludina, Mekongia, and Sinotaia.

The latter group consists of two highly supported clades. Clade 2 comprises Margarya mansuyi from Lakes Xingyun and Qilu, and M. bicotata from Lake Fuxian, which forms the sister group to a Mekongia and Sinotaia subclade. The Margarya subclade within Clade 2 is not highly supported except by BPP values.

Clade 3 comprises all Margarya species from Lake Dianchi and Haixihai reservoir, viz. M. melanioides, $M$. tropidophora, M. monodi and M. bicotata. This clade is not truly a Margarya clade, as it is not even monophyletic for the genus, the species of Margarya clustering indiscriminately with Cipangopaludina dianchiensis and C. sp. Cipangopaludina chinensis and $C$. longispira are also basal or sister, respectively, to all other taxa in Clade 3. The specimens of M. melanioides from Haixihai reservoir are nested within the Lake Dianchi samples, without forming a geographically defined sub-lineage.

At a lower taxonomic level, Margarya bicotata is not monophyletic: the specimen of $M$. bicotata from Lake Fuxian (type locality) is sister group to M. mansuyi in Clade 2 and distinct from those recognized previously as $M$. bi- cotata from Lake Dianchi (Clade 3), which cluster indiscriminately with $M$. melanioides.

\section{Discussion}

The non-monophyly of Margarya, which is shown to comprise three molecular clades, is rather surprising given its rather conspicuous large and ornamented shell that has, until recently, clearly differentiated this genus from all other viviparid genera in East and Southeast Asia. Interestingly, a multiple origin of Margarya was already suggested by Annandale based on differences in transition series from smooth to sculptured shells in the fossil record [15]. The topology of the molecular tree suggests, however, that the comparatively simple shells of Mekongia, Sinotaia and $\mathrm{Ci}$ pangopaludina, which are devoid of any sculpture, have been derived from the unique shell of Margarya, a surprisingly interesting, albeit difficult, fact to interpret. Particularly striking is the fact that species assigned to both Margarya and Cipangopaludina are included in Clade 3 without any support for the monophyly of either (Figure 3). This placement would imply multiple transitions between the two shell forms if the tree were interpreted literally. Shell differences are not only apparent in the adult shells of these two genera, where both share an almost equally large shell size. The embryonic shells also consistently differ, having nodules or spiral keels on the embryonic shell in Margarya as opposed to the three rows of chaetae in Cipangopaludina [39]. The shell may be subjected to environmental pressure and cases of phenotypic plasticity in shell morphology have been reported from various gastropod groups, albeit not from viviparids [20,40,41].

Anatomical data that might provide additional evidence with respect to the molecular results are not available at all for Margarya, and are at best fragmentary for the other Asian genera, Cipangopaludina, Mekongia and Sinotaia, for which our analyses suggest a close relationship to some of the Margarya clades [37,42]. Given this lack of data and the apparent confusion between genera stemming from the sole reliance on a shell-based delineation of taxa, a revision of Margarya and other Asian viviparid genera, particularly 


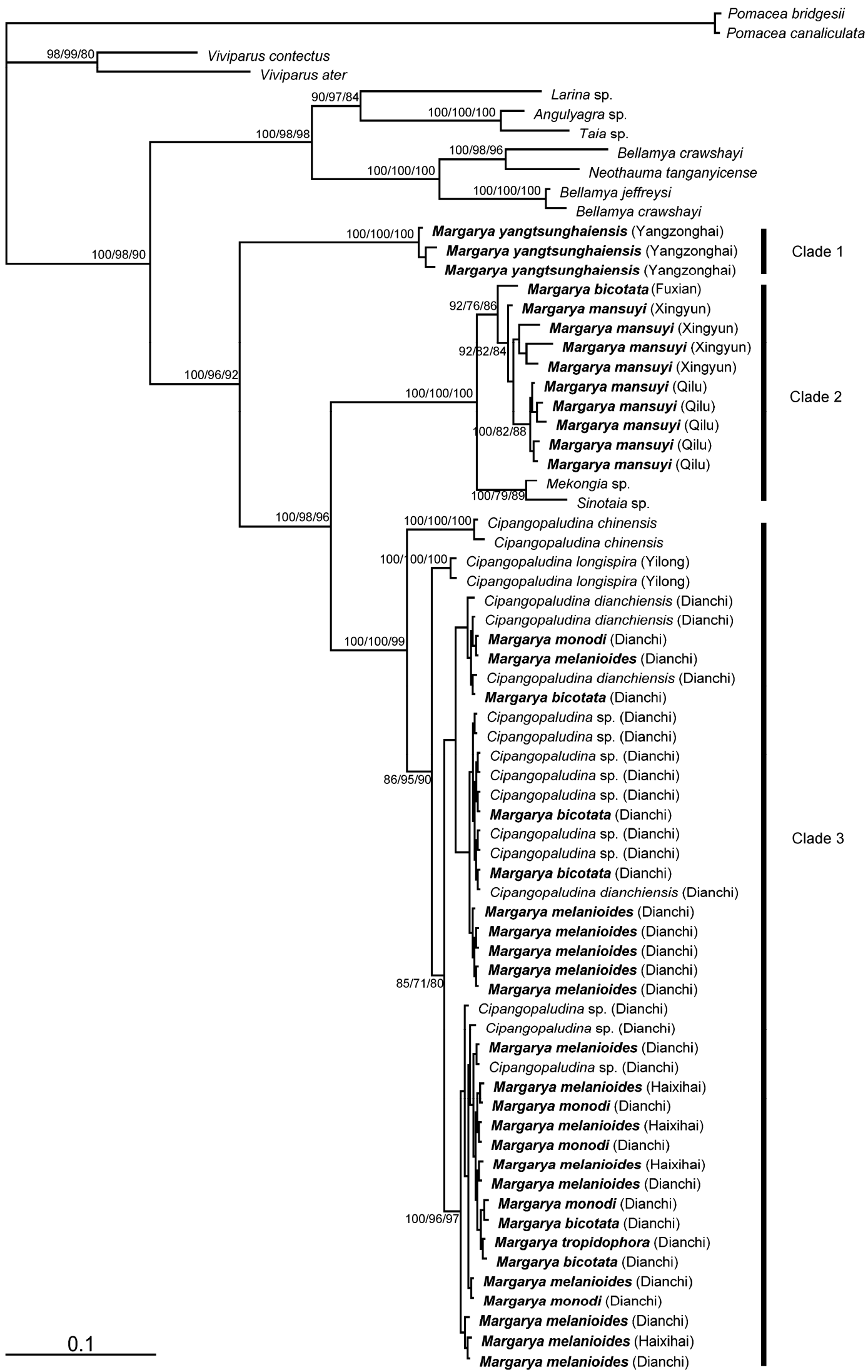

Figure 3 Molecular phylogeny of Margarya based on Bayesian inference. The nodal numbers are BPP, ML and MP bootstrap values in percent. Only values above 50\% are given. The numbered black vertical bars indicate the three major clades (see text) comprising species of Margarya, which are highlighted in bold type. 
Cipangopaludina, Mekongia and Sinotaia, is clearly needed. This need is highlighted by our finding that the monophyly of Cipangopaludina is also not supported. Cipangopaludina chinensis, the type species of Cipangopaludina, and $C$. longispira are paraphyletic with respect to Margarya Clade 3 , and both $C$. dianchiensis and $C$. sp. cluster with several Margarya species in that clade (compare above). The taxonomic confusion at the generic level we found are not exclusive to Asian viviparids, as a recent molecular study on African viviparids found that the characteristic genus Neothauma Smith, 1880 endemic to Lake Tanganyika, is not sister group to the only other African genus Bellamya Jousseaume, 1886, but rather to a subgroup of the latter [24].

Despite the obvious shortcomings of basing genera solely on shell characters, the shell nevertheless provides taxonomically important characters, even in the case of Margarya at an infrageneric level. Shu et al. suggested that $M$. mansuyi (including $M$. bicotata - Clade 2) and M. melanioides (Clade 3) occupy distinct morphospaces as inferred from size ratios [21]. The regular nodules on the entire shell and the pointed apex of $M$. yangtsunghaiensis (Clade 1) distinguish this taxon from all other species of Margarya, and the phylogenetic tree strongly supports this distinctiveness. Data from shell morphology can thus be regarded as compatible with the molecular results with respect to the differences between the three Margarya clades per se, but fail to account for the intergeneric confusion.

At present, drawing any taxonomic conclusion concerning generic assignments from the existing data seems premature. While the non-monophyly of Margarya hints at the possible existence of several genera, it is not possible to tentatively assign the name Margarya to Clade 3, which contains the type species, $M$. melanioides, as its sequences intermittently cluster with those of three other species of Margarya and two species of Cipangopaludina. Specimens from the type locality, which may shed light on this question, were not available at all, as Margarya is more likely than not extinct in Lake Er-hai.

The taxonomy of Margarya is obviously in need of revision at the species level. The only clear pattern at the intrageneric level is the geographic match of the three clades of Margarya with single lakes or lake complexes, viz. (1) Yangzonghai, (2) Fuxian-Xingyun-Qilu, and (3) Dianchi-Haixihai (compare Figure 1). However, with the exception of $M$. yangtsunghaiensis (Clade 1) and M. mansuyi (Clade 2), (sub) clades do not correspond to species of Margarya (Figure 3). This is most striking in Clade 3, where none of the six species occurring in Lake Dianchi and the Haixihai reservoir identified as $M$. melanioides, $M$. tropidophora, M. monodi, M. bicotata, and Cipangopaludina dianchiensis and $C$. sp. appears as monophyletic in the molecular tree. This topology and the low genetic distances (as evidenced by short branch length and low maximum p-distances of 1.6 and $3.2 \%$ for $16 S$ and $C O I$, respectively) within this clade indicate that the previously recognized six species from Lake Dianchi, $M$. melanioides, $M$. tropidophora, M. monodi, M. bicotata, Cipangopaludina dianchiensis, plus $C$. sp., may be conspecific, even though it would remain to be seen in which genus. However, many factors can cause a gene tree to differ from a species tree [43], so we suggest investigating species limits in Margarya by using additional evidence, e.g. from highly variable nuclear markers such as AFLPs or microsatellites, before deciding on the synonymy of species within clades 2 and 3 . It is important to keep in mind that widespread non-monophyly at the species level was also found by Sengupta et al., who also used nuclear markers, in African Bellamya, suggesting that this may be a common phenomenon in viviparids [24].

We greatly thank the "Student Conference on Conservation Science" (University of Cambridge) for supporting several visits by Du LN to Cambridge. We greatly thank Cui GH, Liu C, Li Y and Shu SS for collecting the specimens. We express particular thanks to $Y u$ GH for help with data analysis and Jia L for her help with laboratory work. This research was supported by a GEF Median Size Project (TF051795), Development Marketplace Global Competition Project (DM01333) and Yunnan Provincial Science, Technology Department Social Development Science and Technology Plans (2008CA001) and National Natural Science Foundation of China (30730017).

1 Wang L Z. A research of larger invertebrates in Yunnan Dianchi Lake (in Chinese). J Yunnan Univ, 1985, 7 (Suppl): 73-84

2 Wang L Z. An ecological study on Mollusca population in plateau lakes of Yunnan (in Chinese). J Yunnan Univ, 1988, 10(Suppl), 31: 37-43

3 Huang B Y, Zhang L. Freshwater Lamellibranchia from Dianchi, Yunnan. In: Chinese Society of Malacology, ed. Transactions of the Chinese Society of Malacology, No. 2 (in Chinese). Beijing: Science Press, 1986. 171

4 Huang B Y, Zhang L. The distribution and environment of Unionids from Dianchi and Er-hai Lakes in Yunnan Province. In: Chinese Society of Malacology, ed. Transactions of the Chinese Society of Malacology, No. 3 (in Chinese). Beijing: Science Press, 1990. 69-75

5 Yang J X, Chen Y R. The Biology and Resource Utilization of the Fishes of Fuxian Lake, Yunnan (in Chinese). Kunming: Yunnan Science and Technology Press, 1995

6 Zhang N G, Hao T, Wu C Y, et al. A survey of freshwater gastropod in Yunnan (in Chinese). Stud Marin Sin, 1997, 39: 15-25

7 Cai Y X, Liang X Q. Descriptions of three new species of freshwater shrimps (Crustacea: Decapoda: Atyidae) from Yunnan, southern China. Raff Bul Zool, 1999, 47: 73-80

8 Liang X Q, Cai Y X. Sinodina, a new genus of freshwater shrimps (Crustacea: Decapoda: Atyidae) from southern China, with descriptions of three new species. Raff Bul Zool, 1999, 47: 577-590

9 Chen Z M, Yang J X, Su R F, et al. Present status of the indigenous fishes in Dianchi Lake (in Chinese). Yunnan Biod Sci, 2001, 9: $407-413$

10 Yang L F. The preliminary study on the original classification and distribution law of Lakes on the Yunnan plateau. Trans Oceano Limno, 1984, 1: 34-39

11 Ley S H, Yu M J, Li G Z, et al. Limnological survey of the lakes of Yunnan Plateau. Oceanologia et Limnologia Sini, 1963, 5: 87-114

12 Tchang S, Tsi C Y. A revision of the genus Margarya of the family Viviparidae. Inst Zool Nat Acad Peiping, 1949, 5: 1-25

13 Yen T C. A review of Chinese gastropods in the British museum. Proc Malac Soc London, 1942, 24: 170-289

14 Yen T C. A preliminary revision of the recent species of Chinese 
Viviparidae. The Nautilus, 1943, 56: 124-130

15 Annandale N. The evolution of the shell-sculpture in fresh-water snails of the family Viviparidae. Proc R Soc B, 1924, 96: 60-76

16 Tchang S, Xia W P. The regional differences and the sexual dimorphism of two snails, Margarya melanioides Nevill and Margarya monodi Dautzenberg \& Fischer, from the west coast of Kunming Lake. Inst Zool Nat Acad Peiping, 1949, 5: 67-77

17 Wang S, Xie Y. China Species Red List (Vol. III Invertebrates) (in Chinese). Beijing: Higher Education Press, 2005

18 Tchang S, Cheng C T. Etude sur une paludine comestible, Margarya melanioides, de Tien-Chih. Culture Sino-Francaise, 1945, 1: 1-6

19 Liu Y Y, Zhang W Z, Wang Y X. Geographical distribution of family Viviparidae in China. In: Chinese Society of Malacology, ed. Transactions of the Chinese Society of Malacology, 5-6 (in Chinese). Beijing: Ocean Press, 1995. 8-16

20 Zhang N G, Huang B Y, Cheng Y X. Investigation on ancient snail shell mounts along the shores of Dianchi Lake and Yangzonghai Lake (in Chinese). In: Transactions of the Chinese Society of Malacology, 5-6 (in Chinese). Beijing: Ocean Press, 1995. 178-180

21 Shu F Y, Köhler F, Wang H Z. On the shell and radular morphology of two endangered species of the genus Margarya Nevill, 1877 (Gastropoda: Viviparidae) from lakes of the Yunnan plateau, southwest China. Mollusc Res, 2010, 30: 17-24

22 Chen Y X, Zhang N G. The karyotype study of Margarya yangtsunghaiensis and M. melanioides (Viviparidae). Zool Res, 1996, 17: 94-96

23 Huang X Y, Wang L Z. Analysis of cytochrome oxidase I genes of five species of Margarya and Cipangopaludina chinesis. J Hydroec, 2008, 1: 106-108

24 Sengupta M E, Kristensen T K, Madsen H, et al. Molecular phylogenetic investigations of the Viviparidae (Gastropoda: Caenogastropoda) in the lakes of the Rift Valley area of Africa. Mol Phylogenet Evol, 2009, 52: 797-805

25 Folmer O, Black M, Hoeh W, et al. DNA primers for amplification of mitochondrial cytochrome $c$ oxidase subunit I from diverse metazoan invertebrates. Mol Mar Biol Biotechnol, 1994, 3: 294-299

26 Palumbi S R, Martin A P, Romano S L, et al. The Simple Fool'S Guide to PCR. Hawaii: University of Hawaii, 1991

27 Thompson J D, Gibson T J, Plewniak F, et al. The CLUSTAL X windows interface: Flexible strategies for multiple sequence alignment aided by quality analysis tools. Nucleic Acids Res, 1997, 25:
4876-4882

28 Xia X, Lemey P. Assessing substitution saturation with DAMBE. In: Lemey P, ed. The Phylogenetic Handbook. Cambridge: Cambridge University Press, 2009. 611-626

29 Farris J S, Kallersjo M, Kluge A G, et al. Testing significance of incongruence. Cladistics, 1994, 10: 315-319

30 Swofford D L. PAUP*: Phylogenetic analysis using parsimony (*and Other Methods), Version 4.0. Sinauer Associates, Sunderland, MA. 2002

31 Akaike $\mathrm{H}$. Information theory as an extension of the maximum likelihood principle. In: Petrov B N, Csaki F, eds. Second International Symposium on Information Theory. Budapest: Akademiai Kiado, 1973. 267-281

32 Posada D, Crandall K A. Modeltest: Testing the model of DNA substitution. Bioinformatics, 1998, 14: 817-818

33 Felsenstein J P. Confidence limits on phylogenies: An approach using the bootstrap. Evol, 1985, 39: 783-791

34 Huelsenbeck J P, Bollback J P. Empirical and hierarchical Bayesian estimation of ancestral states. Syst Biol, 2001, 50: 351-366

35 LeachéA D, Reeder T W. Molecular systematics of the eastern fence lizard (Sceloporus undulates): A comparison of parsimony, likelihood, and Bayesian approaches. Syst Biol, 2002, 51: 44-68

36 Huelsenbeck J P, Ronquist F. MrBayes: Bayesian inference of phylogeny. Bioinformatics, 2001, 17: 754-755

37 Rambaut A, Drummond A J. Tracer version 1.0.0. model of DNA substitution. Bioinformatics, 2003, 14: 817-818

38 Kumar S, Tamura K, Nei M. MEGA3: Integrated software for molecular evolutionary genetics analysis and sequence alignment. Bioinformatics, 2004, 5: 150-163

39 Rao H S. On the comparative anatomy of oriental Viviparidae. Rec Indian Mus, 1925, 27: 129-135

40 Palmer A R. Quantum changes in gastropod shell morphology need not reflect speciation. Evolution, 1985, 39: 699-705

41 Trussell G C, Smith C D. Induced defenses in response to an invading crab predator: An explanation of historical and geographic phenotypic change. Proc Nat Acad Sci USA, 2000, 97: 2123-2127

42 Taki I. Anatomical study of Cipangopaludina migagii, Kuroda. Venus, 1941, 16: 131-134

43 Funk D J, Omland K E. Species-level paraphyly and polyfyly: Frequency, causes and consequences, with insights from animal mitochondrial DNA. Annu Rev Ecol Syst, 2003, 34: 397-423

Open Access This article is distributed under the terms of the Creative Commons Attribution License which permits any use, distribution, and reproduction in any medium, provided the original author(s) and source are credited. 